\title{
Norma do sup para equações de advecção-difusão duplamente não lineares: um caso de decrescimento
}

Jocemar de Q. Chagas ${ }^{1}$

Departamento de Matemática e Estatística, UEPG, Ponta Grossa, PR

Patrícia L. Guidolin ${ }^{2}$

Instituto Federal de Ciência, Educação e Tecnologia do Rio Grande do Sul, IFRS, Farroupilha, RS Paulo R. Zingano ${ }^{3}$

Departamento de Matemática Pura e Aplicada, UFRGS, Porto Alegre, RS

Resumo. Neste trabalho usaremos uma série de desigualdades de energia padrão para implementar um refinado argumento do tipo $L^{p}-L^{q}$, visando obter, de uma forma relativamente curta, a derivação da seguinte estimativa fundamental na norma do sup:

$$
\|u(\cdot, t)\|_{L^{\infty}\left(\mathbb{R}^{n}\right)} \leq K(n, p)\left\|u_{0}\right\|_{L^{p}\left(\mathbb{R}^{n}\right)}^{\rho} t^{\sigma}, \quad \forall t>0,
$$

onde $\rho=\frac{p(\beta+2)}{n(\alpha+\beta)+p(\beta+2)}$ e $\sigma=\frac{n}{n(\alpha+\beta)+p(\beta+2)}$, para soluções da equação de adveção-difusão duplamente não linear regularizada

$$
u_{t}+\operatorname{div}(f(x, t, u))=\operatorname{div}\left(|u|^{\alpha}|\nabla u|^{\beta} \nabla u\right)+\eta \Delta u,
$$

quando $f$ atende a determinadas condições, expostas no texto a seguir.

Palavras-chave. Equações Diferenciais Parciais, Equações de Evolução, Equações Parabólicas, Estimativas para a norma do sup, Soluções Globais.

\section{Introdução}

Consideraremos o problema regularizado

$$
\left\{\begin{array}{l}
u_{t}+\operatorname{div}(\boldsymbol{f}(x, t, u))=\operatorname{div}\left(|u|^{\alpha}|\nabla u|^{\beta} \nabla u\right)+\eta \Delta u, \quad x \in \mathbb{R}^{n}, t>0, \\
u(\cdot, 0)=u_{0} \in L^{p_{0}}\left(\mathbb{R}^{n}\right) \cap L^{\infty}\left(\mathbb{R}^{n}\right),
\end{array}\right.
$$

onde $\eta>0$ está fixo e $1 \leq p_{0}<\infty$ é dado; $\alpha$ e $\beta$ são constantes, com $\alpha, \beta \geq 0$ e $\alpha+\beta>0$; e a função $\boldsymbol{f}(x, t, u)$ satisfaz $|\boldsymbol{f}(x, t, u)| \leq B(T)|u|^{\kappa+1}$, para $\kappa \geq 0, \forall x \in \mathbb{R}^{n}, t \geq 0$, $u \in \mathbb{R},\left(B(T)<\infty\right.$ denota a variação de $\boldsymbol{f}(x, t, u)$ em $\mathbb{R}^{n}$, e controla o tamanho de suas derivadas) e a condição de estabilidade

$$
\sum_{i=1}^{n} u \frac{\partial \boldsymbol{f}}{\partial x_{i}}(x, t, u) \geq 0
$$

\footnotetext{
${ }^{1}$ jocemarchagas@uepg.br

${ }^{2}$ patricia.guidolin@ifrs.edu.br

${ }^{3}$ paulo.zingano@ufrgs.br
} 
e apresentaremos as ideias que permitem obter para suas soluções a seguinte estimativa fundamental na norma do sup:

$$
\|u(\cdot, t)\|_{L^{\infty}\left(\mathbb{R}^{n}\right)} \leq K(n, p)\left\|u_{0}\right\|_{L^{p}\left(\mathbb{R}^{n}\right)}^{\rho} t^{-\sigma}, \quad \forall t>0,
$$

bem como estipular os valores de $\rho$ e $\sigma$ para os quais a estimativa é válida.

A existência de solução suave para o problema regularizado (1) em um determinado intervalo $\left[0, T_{*}\right.$ ) é garantida pela teoria geral de equações parabólicas (ver, por exemplo, [6]). Estimativas do tipo (3) podem também ser obtidas por outros métodos, como desigualdades de Sobolev logarítmicas ou técnicas de Fourier Splitting (ver, por exemplo, [3] e [8]). As ideias apresentadas neste trabalho podem ser vistas com mais detalhes, por exemplo, em [1], onde estão aplicadas a uma equação um pouco mais simples; em [2] e em [5].

A seguir, enunciaremos os principais resultados a serem percorridos no caminho de se obter (3), além de dar uma breve ideia de cada demonstração.

\section{Resultados}

O primeiro resultado a ser apresentado diz que a norma $L^{q}$ das soluções suaves $u(\cdot, t)$ decresce, à medida que $t$ cresce.

Teorema 2.1. Seja $u(\cdot, t) \in L^{\infty}\left([0, T], L^{\infty}\left(\mathbb{R}^{n}\right)\right)$ solução suave do problema (1), para algum $0<T<T_{*}$, onde $\boldsymbol{f}(x, t, u)$ satisfaz a condição (2). Então, para cada $p_{0} \leq q \leq \infty$, vale

$$
\|u(\cdot, t)\|_{L^{q}\left(\mathbb{R}^{n}\right)} \leq\left\|u_{0}\right\|_{L^{q}\left(\mathbb{R}^{n}\right)}, \quad \forall 0<t \leq T
$$

Ideia da prova: Consideramos $p_{0} \leq q<\infty$ e, para $\delta>0, R>0$ e $0<\varepsilon \leq 1$ dados, multiplicamos a equação em (1) por $\Phi_{\delta}^{\prime}(u) \cdot \zeta_{R}(x)$, onde $\Phi_{\delta}(u)$ é a função auxiliar definida por $\Phi_{\delta}(u):=u^{2}$, se $q=2$; ou $\Phi_{\delta}(u):=\left(L_{\delta}(u)\right)^{q}$, se $q>2$, onde $L_{\delta}(u):=\int_{0}^{u} S\left(\frac{v}{\delta}\right) d v$ e $S(v)$ é tal que $S^{\prime}(v) \geq 0 \forall v, S(0)=0$ e $S(v)=\operatorname{sgn}(v),|v| \geq 1$; e $\zeta_{R}(x)$ é a função de corte definida por $\zeta_{R}(x):=e^{-\varepsilon \sqrt{1+|x|^{2}}}-e^{-\varepsilon \sqrt{1+R^{2}}}$, se $|x| \leq R$; ou $\zeta_{R}(x):=0$, se $|x|>R$.

Após integrar a equação resultante

$$
\begin{aligned}
& \Phi_{\delta}^{\prime}(u) u_{t} \zeta_{R}(x)+\Phi_{\delta}^{\prime}(u) \operatorname{div}(\boldsymbol{f}(x, t, u)) \zeta_{R}(x)= \\
& \quad=\Phi_{\delta}^{\prime}(u) \mu(t) \operatorname{div}\left(|u|^{\alpha}|\nabla u|^{\beta} \nabla u\right) \zeta_{R}(x)+\Phi_{\delta}^{\prime}(u) \eta \Delta u \zeta_{R}(x)
\end{aligned}
$$

sobre $\mathbb{R}^{n} \times\left[t_{0}, t\right]$, com $0<t_{0}<t \leq T$, rearranjar adequadamente os termos, e efetuar as passagens ao limite com $\delta \rightarrow 0$ e $R \rightarrow \infty$, chegamos a

$$
U_{\varepsilon}(t) \leq U_{\varepsilon}(0)+\varepsilon C_{1}(T) \int_{0}^{t} U_{\varepsilon}(\tau) d \tau+\varepsilon C_{2}(T) \int_{0}^{t} \mu(\tau) U_{\varepsilon}(\tau) d \tau
$$

onde $U_{\varepsilon}(t):=\int_{\mathbb{R}^{n}}|u(x, t)|^{q} e^{-\varepsilon \sqrt{1+|x|^{2}}} d x$, para $q \geq 2$, e $C_{1}(T), C_{2}(T)$ são constantes dependentes apenas de $n, \eta, B(T)$ e $M(T)$, com $M(T) \geq\|u(\cdot, t)\|_{L^{\infty}\left(\mathbb{R}^{n}\right)} \forall 0 \leq t \leq T$. 
Ao aplicar o Lema de Gronwall a (4) e passar ao limite com $\varepsilon \rightarrow 0$, obtemos

$$
\|u(\cdot, t)\|_{L^{q}\left(\mathbb{R}^{n}\right)} \leq\|u(\cdot, 0)\|_{L^{q}\left(\mathbb{R}^{n}\right)}, \quad \forall 0 \leq t \leq T,
$$

para $q$ satisfazendo a $q \geq 2$ e $p_{0} \leq q<\infty$

Então, simplesmente fazendo $q \rightarrow \infty$, concluímos que

$$
\|u(\cdot, t)\|_{L^{\infty}\left(\mathbb{R}^{n}\right)} \leq\|u(\cdot, 0)\|_{L^{\infty}\left(\mathbb{R}^{n}\right)}, \quad \forall 0 \leq t \leq T .
$$

A demonstração para o caso $1<q<2$ segue os mesmos procedimentos, porém deve-se efetuar a passagem ao limite $\operatorname{com} \delta \rightarrow 0$ por último.

A desigualdade indicada no Lema 2.1 será utilizada adiante para derivar estimativas de controle para a solução.

Lema 2.1. Seja $u(\cdot, t) \in L_{\text {loc }}^{\infty}\left([0, \infty), L^{\infty}\left(\mathbb{R}^{n}\right)\right)$ solução suave do problema (1). Se $\boldsymbol{f}(x, t, u)$ satisfaz a condição (2), entẫo, $\forall 0<t_{0}<t$ e $\gamma>0$, podemos obter a seguinte desigualdade:

$$
\begin{gathered}
\left(t-t_{0}\right)^{\gamma}\|u(\cdot, t)\|_{L^{q\left(\mathbb{R}^{n}\right)}}^{q}+q(q-1) \int_{t_{0}}^{t}\left(\tau-t_{0}\right)^{\gamma} \int_{\mathbb{R}^{n}}|u(x, \tau)|^{q-2+\alpha}|\nabla u(x, \tau)|^{\beta+2} d x d \tau \leq \\
\leq \gamma \int_{t_{0}}^{t}\left(\tau-t_{0}\right)^{\gamma-1}\|u(\cdot, \tau)\|_{L^{q}\left(\mathbb{R}^{n}\right)}^{q} d \tau .
\end{gathered}
$$

Ideia da prova: O Lema 2.1 tem demonstração similar ao Teorema 2.1, mas no início multiplicamos a equação em (1) por $\left(t-t_{0}\right)^{\gamma} \cdot \Phi_{\delta}^{\prime}(u) \cdot \zeta_{R}(x)$, onde o expoente $\gamma$ deve ser adequadamente escolhido mais tarde.

O próximo resultado indica que a norma $L^{q}$ da solução, em um intervalo de tempo, pode ser controlada por uma norma mais baixa, computada na extremidade esquerda do intervalo.

Teorema 2.2. Seja $u(\cdot, t) \in L_{\text {loc }}^{\infty}\left([0, \infty), L^{\infty}\left(\mathbb{R}^{n}\right)\right)$ solução suave do problema (1). Se $f(x, t, u)$ satisfaz a condição (2), então podemos obter:

$$
\|u(\cdot, t)\|_{L^{q}\left(\mathbb{R}^{n}\right)} \leq K_{q}(n, \alpha, \beta)\left\|u\left(\cdot, t_{0}\right)\right\|_{L^{q / 2}\left(\mathbb{R}^{n}\right)}^{\rho}\left(t-t_{0}\right)^{-\sigma},
$$

onde $\rho=\frac{q(\beta+2)+n(\alpha+\beta)}{q(\beta+2)+2 n(\alpha+\beta)}$ e $\sigma=\frac{n}{q(\beta+2)+2 n(\alpha+\beta)}$, para qualquer $t_{0}<t$ e $2 p_{0} \leq q<\infty$.

Ideia da prova: Começamos definindo $w(\cdot, t) \in L^{1}\left(\mathbb{R}^{n}\right) \cap L^{\infty}\left(\mathbb{R}^{n}\right)$ por

$$
w(x, t):=|u(x, t)|^{\lambda_{1}}, \quad \forall x \in \mathbb{R}^{n}, \forall t>0,
$$

onde $\lambda_{1} \geq 1$ e $u \in L^{p}\left(\mathbb{R}^{n}\right)$ é solução do problema (1), com $\boldsymbol{f}(x, t, u)$ satisfazendo a condição (2). Com o objetivo inicial de reescrever a equação (5) em termos de $w$, ao analisar o $2^{\circ}$ 
termo, somos levados a escolher $\lambda_{1}=\frac{q+\alpha+\beta}{(\beta+2)}$. Ao reescrever a equação (5) em termos de $w$ encontramos

$$
\begin{gathered}
\left(t-t_{0}\right)^{\gamma}\|w(\cdot, t)\|_{L^{\lambda}\left(\mathbb{R}^{n}\right)}^{\lambda}+q(q-1)\left(\frac{(\beta+2)}{q+\alpha+\beta}\right)^{(\beta+2)} \int_{t_{0}}^{t}\left(\tau-t_{0}\right)^{\gamma} \mu(\tau)\|\nabla w(\cdot, \tau)\|_{L^{\beta+2}\left(\mathbb{R}^{n}\right)}^{\beta+2} d \tau \leq \\
\leq \gamma \int_{t_{0}}^{t}\left(\tau-t_{0}\right)^{\gamma-1}\|w(\cdot, \tau)\|_{L^{\lambda}\left(\mathbb{R}^{n}\right)}^{\lambda} d \tau,
\end{gathered}
$$

onde $\lambda=\frac{q(\beta+2)}{q+\alpha+\beta}$ é tomado de forma que $\|u(\cdot, t)\|_{L^{q}\left(\mathbb{R}^{n}\right)}^{q}=\|w(\cdot, t)\|_{L^{\lambda}\left(\mathbb{R}^{n}\right)}^{\lambda}, \forall t>0$.

A seguir, usamos em (7) a seguinte desigualdade de interpolação do tipo SobolevNirenberg-Gagliardo (SNG):

$$
\|w(\cdot, t)\|_{L^{r}\left(\mathbb{R}^{n}\right)} \leq C\|w(\cdot, t)\|_{L^{s}\left(\mathbb{R}^{n}\right)}^{1-\theta}\|\nabla w(\cdot, t)\|_{L^{p}\left(\mathbb{R}^{n}\right)}^{\theta}, \quad \forall w \in C_{0}^{1}\left(\mathbb{R}^{n}\right),
$$

onde $0 \leq \theta \leq 1$, e $r, s$ e $p$ satisfazem a $0<s \leq r \leq \infty, 1 \leq p \leq \infty$, e $\frac{1}{r}=\left(\frac{1}{p}-\frac{1}{n}\right) \theta+\frac{(1-\theta)}{s}$. (mais sobre essa desigualdade pode ser encontrado, por exemplo, em [4] ou em [7]). Com os valores $r=\lambda, s=\frac{\lambda}{2}$ e $p=(\beta+2)$, a desigualdade (8) toma a forma

$$
\|w(\cdot, t)\|_{L^{\lambda}\left(\mathbb{R}^{n}\right)} \leq C\|w(\cdot, t)\|_{L^{\lambda / 2}\left(\mathbb{R}^{n}\right)}^{1-\theta}\|\nabla w(\cdot, t)\|_{L^{\beta+2}\left(\mathbb{R}^{n}\right)}^{\theta},
$$

onde $\theta=\frac{n(q+\alpha+\beta)}{n q+q(\beta+2)+2 n(\alpha+\beta)}$.

Após alguns procedimentos, incluindo o uso do Teorema 2.1 e a escolha de $\gamma$ como $\gamma=\frac{(\beta+2)}{(\beta+2)-\theta \lambda}$, obtemos

$$
\|w(\cdot, t)\|_{L^{\lambda}\left(\mathbb{R}^{n}\right)}^{\lambda} \leq K_{1}(q, n, \alpha, \beta)\left\|u\left(\cdot, t_{0}\right)\right\|_{L^{q / 2}\left(\mathbb{R}^{n}\right)}^{\frac{q(1-\theta)(\beta+2)}{(\beta+2)-\theta \lambda}}\left(t-t_{0}\right)^{1-\gamma} .
$$

Ao reescrevermos o lado esquerdo de (9) em termos de $u$, obtemos a expressão desejada $(6), \operatorname{com} K_{q}(n, \alpha, \beta)=\left(K_{1}(q, n, \alpha, \beta)\right)^{1 / q}, \rho=\frac{(1-\theta)(\beta+2)}{(\beta+2)-\theta \lambda}$ e $\sigma=\frac{\gamma-1}{q}=\frac{n}{q(\beta+2)+2 n(\alpha+\beta)}$.

Por fim, e como resultado principal, mostramos que para todo $t>t_{0}$ a norma $L^{\infty}$ da solução é majorada por uma função positiva, que decresce à medida que $t$ aumenta.

Teorema 2.3. Seja $u(\cdot, t) \in L_{l o c}^{\infty}\left([0, \infty), L^{\infty}\left(\mathbb{R}^{n}\right)\right)$ solução suave do problema (1). Se $\boldsymbol{f}(x, t, u)$ satisfaz a condição (2), então podemos obter:

$$
\|u(\cdot, t)\|_{L^{\infty}\left(\mathbb{R}^{n}\right)} \leq K(n, \alpha, \beta, q)\left\|u\left(\cdot, t_{0}\right)\right\|_{L^{q\left(\mathbb{R}^{n}\right)}}^{\rho}\left(t-t_{0}\right)^{-\sigma}
$$

para qualquer $t_{0}<t$ e $2 p_{0} \leq q<\infty$, onde $\rho=\frac{q(\beta+2)}{n(\alpha+\beta)+q(\beta+2)}$ e $\sigma=\frac{n}{n(\alpha+\beta)+q(\beta+2)}$.

Quando $t_{0}=0,(10)$ se torna a estimativa desejada (3). Além disso, vale ressaltar que os valores de $\rho$ e $\sigma$ apresentados no Teorema 2.3 são compatíveis com análise de escalas.

Ideia da prova: Para provar (10), usamos um procedimento iterativo: buscamos estimar $\|u(\cdot, t)\|_{L^{\infty}\left(\mathbb{R}^{n}\right)}$ num instante $t$, com $0 \leq t_{0}<t$, em termos de um valor conhecido 
$\left\|u\left(\cdot, t_{0}\right)\right\|_{L^{p}\left(\mathbb{R}^{n}\right)}$, onde $u(\cdot, t) \in L^{p}\left(\mathbb{R}^{n}\right) \cap L^{\infty}\left(\mathbb{R}^{n}\right)$. Para isso, tomamos $m>1$ fixo e estimamos inicialmente $\left\|u\left(\cdot, t_{m}=t\right)\right\|_{L^{2^{m_{p}}\left(\mathbb{R}^{n}\right)}}$ em termos de um tempo anterior $t_{m-1}$ na norma $L^{2^{(m-1)} p}$. Depois, estimamos $\left\|u\left(\cdot, t_{m-1}\right)\right\|_{L^{2(m-1)} p\left(\mathbb{R}^{n}\right)}$ em termos de um tempo anterior $t_{m-2}$ na norma $L^{2^{(m-2)} p}$, e assim sucessivamente, até chegar ao instante de tempo inicial $t_{0}$. Ao proceder dessa maneira conseguimos estimar $\|u(\cdot, t)\|_{L^{2^{m}} p_{\left(\mathbb{R}^{n}\right)}}$ em termos do valor conhecido $\left\|u\left(\cdot, t_{0}\right)\right\|_{L^{p}\left(\mathbb{R}^{n}\right)}$, e então podemos obter o resultado desejado ao fazer $m \rightarrow \infty$.

Para $m>1$ fixo, particionamos o intervalo de tempo $\left(t_{0}, t\right)$ em partes disjuntas e sequenciais, fazendo:

$$
\left\{\begin{array}{l}
t_{0}^{(m)}=t_{0}+2^{-m}\left(t-t_{0}\right) \\
t_{i}^{(m)}=t_{0}^{(m)}+\left(1-2^{-i}\right)\left(t-t_{0}\right), \quad \forall 1 \leq i \leq m-1 ; \quad \mathrm{e} \\
t_{m}^{(m)}=t_{0}^{(m)}+\left(1-2^{-m}\right)\left(t-t_{0}\right)=t .
\end{array}\right.
$$

Cosidere inicialmente $q=2^{m}(p)$. Pelo Teorema 2.2, para qualquer $t_{0}<t$ e $2 p \leq q<\infty$, é válida a estimativa

$$
\|u(\cdot, t)\|_{L^{q}\left(\mathbb{R}^{n}\right)} \leq K_{q}(n, \alpha, \beta)\left\|u\left(\cdot, t_{0}\right)\right\|_{L^{q / 2}\left(\mathbb{R}^{n}\right)}^{\frac{q(\beta+2)+n(\alpha+\beta)}{q(\beta+2)+2 n(\alpha+\beta)}}\left(t-t_{0}\right)^{\frac{-n}{q(\beta+2)+2 n(\alpha+\beta)}} .
$$

Usando (11) para estimar $\left\|u\left(\cdot, t_{m}^{(m)}\right)\right\|_{L^{2^{m} p_{\left(\mathbb{R}^{n}\right)}}}$ em termos de $\left\|u\left(\cdot, t_{m-1}^{(m)}\right)\right\|_{L^{2^{(m-1)} p\left(\mathbb{R}^{n}\right)}}$, obtemos

$$
\left\|u\left(\cdot, t_{m}^{(m)}\right)\right\|_{L^{2^{m} p\left(\mathbb{R}^{n}\right)}} \leq K_{m}\left\|u\left(\cdot, t_{m-1}^{(m)}\right)\right\|_{L^{2^{(m-1)} p\left(\mathbb{R}^{n}\right)}}^{\left(\frac{2^{m} p(\beta+2)+n(\alpha+\beta)}{2^{m} p(\beta+2)+2 n(\beta)}\right)}\left(t_{m}^{(m)}-t_{m-1}^{(m)}\right)\left(\frac{-n}{2^{m} p(\beta+2)+2 n(\alpha+\beta)}\right) .
$$

A seguir usamos (11) para estimar $\left\|u\left(\cdot, t_{m-1}^{(m)}\right)\right\|_{L^{2^{(m-1)} p\left(\mathbb{R}^{n}\right)}}$ em termos de $t_{m-2}^{(m)}$.

Repetindo esse procedimento para cada $1 \leq i \leq m$, chegamos a

$$
\left\|u\left(\cdot, t_{m}^{(m)}\right)\right\|_{L^{2^{m} p\left(\mathbb{R}^{n}\right)}} \leq \prod_{i=1}^{m}\left(K_{i}^{A_{m-i}} \cdot\left\|u\left(\cdot, t_{0}^{(m)}\right)\right\|_{L^{p}\left(\mathbb{R}^{n}\right)}^{B_{m}} \cdot\left(2^{-i}\left(t-t_{0}\right)\right)\left(\frac{-n}{2^{i} p(\beta+2)+2 n(\alpha+\beta)} \cdot A_{m-i}\right)\right),
$$

onde $A_{0}:=1 ; \forall 1 \leq i \leq m-1$ tem-se $A_{i}:=\prod_{j=0}^{i-1}\left(\frac{2^{m-j} p(\beta+2)+n(\alpha+\beta)}{2^{m-j} p(\beta+2)+2 n(\alpha+\beta)}\right) ; \mathrm{e}, \forall 1 \leq i \leq m$, $B_{i}:=\prod_{j=m+1-i}^{m}\left(\frac{2^{j} p(\beta+2)+n(\alpha+\beta)}{2^{j} p(\beta+2)+2 n(\alpha+\beta)}\right)$.

O próximo procedimento é analisar o comportamento de cada um dos fatores que compõem o lado direito de (12), quando $m \rightarrow \infty$.

Analisando $\prod_{i=1}^{m}\left(2^{-i}\left(t-t_{0}\right)\right)^{\left(\frac{-n}{2^{i} p(\beta+2)+2 n(\alpha+\beta)} \cdot A_{m-i}\right)}$ ao $m \rightarrow \infty$, descobrimos que a potência de $\left(t-t_{0}\right)$ se torna $\frac{n}{p(\beta+2)+n(\alpha+\beta)}$, ou seja, $\sigma$, e que a constante é finita.

Ao efetuar a passagem ao limite com $m \rightarrow \infty$ no expoente $B_{m}$ que aparece em (12), descobrimos que $\lim _{m \rightarrow \infty} B_{m}=\frac{p(\beta+2)}{p(\beta+2)+n(\alpha+\beta)}$, que é o valor esperado para $\rho$.

Finaliza-se a demonstração mostrando, com algum trabalho, que ao fazer $m \rightarrow \infty$ o produtório $\prod_{i=1}^{m} K_{i}^{A_{m-i}}$ que aparece em (12) é finito. 


\section{Conclusões}

Sobre as soluções do problema (1), onde $\boldsymbol{f}(x, t, u)$ satisfaz a condição (2), apresentamos no Teorema 2.1 um resultado de decrescimento da norma $L^{q}$ da solução $u(\cdot, t)$, para cada $p_{0} \leq q \leq \infty$ e $\forall 0<t \leq T<T_{*}$. Como consequência direta daquele resultado, podemos concluir que a solução é global (ou seja, é definida para todo $t>0$ ). A estimativa de decrescimento (3), nosso principal resultado, é obtida no Teorema 2.3. Como resultado final, como a solução é definida para qualquer $t>0$, podemos concluir, usando os Teoremas 2.1 e 2.3 , que a solução vai a zero ao $t \rightarrow \infty$.

\section{Referências}

[1] P. Braz e Silva, L. Schütz and P. R. Zingano. On some energy inequalities and supnorm estimates for advection-diffusion equations in $\mathbb{R}^{n}$, Nonlinear Analysis: Theory, Methods \& Applications, v. 93, 90-96, 2013. DOI: 10.1016/j.na.2013.07.028

[2] J. Q. Chagas. Contribuições para a teoria de equações parabólicas duplamente não lineares com termos advectivos. Tese de Doutorado, UFRGS, 2015.

[3] M. Escobedo and E. Zuazua. Large time behavior for convection-diffusion equations in $\mathbb{R}^{n}$, J. Funct. Anal., 100, p. 119-161, 1991.

[4] A. Friedman. Partial Differential Equations, Holt, Rinehart and Winston, New York, 1969.

[5] P. L. Guidolin. Contribuições para a teoria de equações do p-Laplaciano evolutivo com termos advectivos. Tese de Doutorado, UFRGS, 2015.

[6] O. A. Ladyzhenskaya, V. A. Solonnikov and N. N. Uralceva. Linear and Quasilinear Equations of Parabolic Type, American Mathematical Society, Providence, 1968.

[7] L. Nirenberg. On elliptic partial differential equations. Annali della Scuola Normale Superiore di Pisa, Classe di Scienze $3^{\mathrm{a}}$ série, tome 13, no 2, p. 115-162, 1959.

[8] M. M. Porzio. On decay estimates, J. Evol. Eqs., 9, p. 561-591, 2009. 